\title{
Microbial and sensory evaluation of ohmic heat processed mango juice
}

\author{
V. Perasiriyan, S. Priya and A. Mangala Gowri
}

\begin{abstract}
Development of new technologies in thermal food treatments are showing promise for industrial and scientific processing of foods with minimum nutrient loss. Fruits and their products have gained considerable importance by contributing significantly to the economy of India, the second largest producer of fruits and vegetables in the world. Food quality preservation through ohmic heating processing (OHP) technology is useful for the treatment of protein rich perishable foods like fruits which tends to denature and coagulate when thermally processed. Microbial inactivation in relation to ohmic heating is primarily thermal in nature, much like conventional heating. At low frequencies $(50-60 \mathrm{~Hz})$ and high field strengths, charges were built upon the porous cell walls of the micro-organism. The thermal pasteurization and OHP exhibited in the present study significant reduction in microbial load and prolonged shelf-life of mango juice. The study results obtained support the use of OHP to improve the quality of mango juice along with safety standards as an alternative to thermal pasteurization.
\end{abstract}

Key Words : Ohmic heat process, Mango juice, Microbial, Sensory

How to cite this article : Perasiriyan, V., Priya, S. and Gowri, A. Mangala (2019). Microbial and sensory evaluation of ohmic heat processed mango juice. Food Sci. Res. J., 10(1): 101-104, DOI : 10.15740/HAS/FSRJ/10.1/101-104. Copyright@ 2019: Hind AgriHorticultural Society.

A. Mangala Gowri, Centre for Stem Cell Research and Regenerative Medicine, Madras Veterinary College, Tamil Nadu Veterinary and Animal 